\title{
The Multi Angle Polarimeter (MAP) on board ESA's Copernicus Carbon Dioxide Monitoring mission (CO2M)
}

David Spilling, Andrew Thales

David Spilling, Andrew Thales, "The Multi Angle Polarimeter (MAP) on board ESA's Copernicus Carbon Dioxide Monitoring mission (CO2M)," Proc. SPIE 11852, International Conference on Space Optics — ICSO 2020, 118520R (11 June 2021); doi: 10.1117/12.2599174

SPIE Event: International Conference on Space Optics - ICSO 2021, 2021, Online Only 


\section{International Conference on Space Optics-ICSO 2020}

Virtual Conference

30 March-2 April 2021

Edited by Bruno Cugny, Zoran Sodnik, and Nikos Karafolas
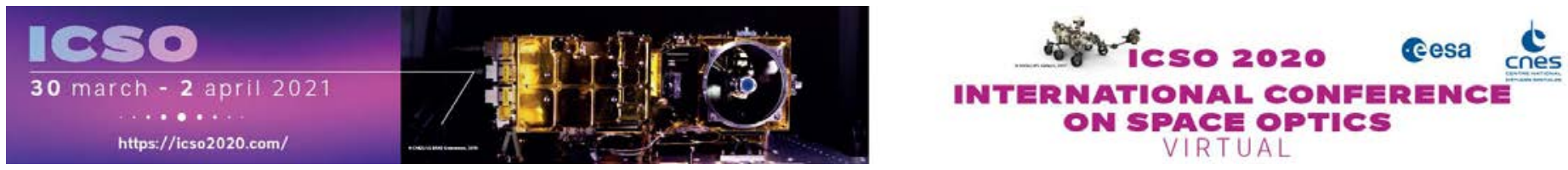

\section{The Multi Angle Polarimeter (MAP) on board ESA's Copernicus Carbon Dioxide Monitoring mission (CO2M)}

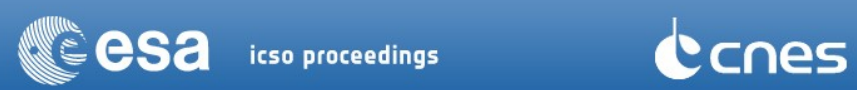




\title{
The Multi Angle Polarimeter (MAP) on board ESA's Copernicus Carbon Dioxide Monitoring mission (CO2M)
}

\author{
David Spilling $*^{\mathrm{a}}$, Andrew Walker ${ }^{\mathrm{b}}$ \\ ${ }^{\mathrm{a}}$ Thales Alenia Space, Bristol, UK; ${ }^{\mathrm{b}}$ Teledyne e2v, Chelmsford, UK
}

\begin{abstract}
The $\mathrm{CO} 2 \mathrm{M}$ mission, part of the Copernicus Expansion programme, will measure anthropogenic $\mathrm{CO} 2$. Due to the accuracy requirements of this measurement, it is important to measure and characterise the aerosol population within the $\mathrm{CO} 2$ instrument footprint. For this purpose, ESA have requested that a Multi Angle Polarimeter (MAP) be part of the instrument suite.

As part of the overall mission consortium led by OHB (DE), Thales Alenia Space (UK) have been selected to provide this instrument. The instrument itself is a multi angle ( $>40$ angles) multi spectral ( 7 bands, 400-900nm) polarimeter, based on well known approaches (POLDER, 3MI). However, MAP achieves the demanding level of accuracy in a compact volume by using a focal plane assembly that performs both polarization and spectral separation. Use of simple telescopes also allows for wide field-of-view $(300 \mathrm{~km}$ swath) while minimising issues associated with straylight.

This paper outlines the main MAP instrument requirements, and details the instrument operating concept and design. Results from the early technology predevelopment activity will be presented, as well as a brief look at other missions for which MAP is suited.
\end{abstract}

Keywords: Imaging Polarimeter, Aerosol, Micropolarizer, Copernicus, Sentinel 7

\section{INTRODUCTION}

The CO2M mission will provide the European Union with an operational capacity that contributes to the global monitoring of anthropogenic $\mathrm{CO} 2$ emissions. The primary objective is to measure images of total column $\mathrm{CO} 2$ with the resolution, accuracy, time sampling and spatial coverage required to provide the key space component at the scale of cities, large industrial sites, regions, countries, and the Earth as a whole.

Differentiation of anthropogenic $\mathrm{CO} 2$ from other sources requires a high degree of accuracy in the retrieval; a systematic bias error better than $0.5 \mathrm{ppm}$ in $\mathrm{XCO} 2$ is required [1]. Aerosol scattering effects can degrade this retrieval accuracy, leading to systematic error, consequently knowledge of the aerosol field is important and this will be obtained through measurements performed by a Multi Angle Polarimeter (MAP).

Phase A/B1 of the CO2M project commenced in 2018, with two parallel industrial studies. Thales Alenia Space (TAS) in the UK were chosen to provide the MAP, as part of one industrial consortium, working with TAS in France who are the prime for the Payload module, and who also lead the development of the main $\mathrm{CO} 2 \& \mathrm{NO} 2$ spectrometers.

At the start of the project, the MAP was seen as an auxiliary payload, which was not necessarily going to be mandated as part of the B2/C/D payload. TAS' approach was therefore to aim for as compact an instrument as possible, in order to reduce the resource requirements on the platform and make it more likely to fly. In fact, during the Phase A/B1 program, the aerosol retrieval function evolved from the status of a potential auxiliary requirement, and hence payload, to a musthave, and this was reflected in the ITT for the Phase B2/C/D.

As will be shown later, the TAS instrument is built on well known retrieval techniques, but with some technological innovation to reduce the size. The new innovation was the subject of successful targeted predevelopment during the Phase A/B1, which demonstrated that the performance could be met. 


\section{INSTRUMENT REQUIREMENTS}

The two driving instrument requirements are the viewing geometry, and the precision of retrieval.

Retrieval of aerosol parameters is achieved by multiangular, multispectral, observations of the radiance and degree of linear polarization of light scattered from the aerosol particles, where the observation angles are spread between $-60^{\circ}$ zenith angle (OZA) to $+60^{\circ} \mathrm{OZA}$. Within that range, there is a tradeoff between the number of angles and number of spectral bands, and the current range of polarimeters have chosen different points in this continuum. The mission requirements admitted for several solutions; the one proposed by TAS favored a reduced number of spectral channels but a large number of retrieved view angles. Here a "view" is the ensemble of all spectral and polarization measurements at a particular OZA.

Table 1. Spectral channels and number of angular views for several current polarimeters.

\begin{tabular}{|c|l|c|}
\hline $\begin{array}{c}\text { Instrument } \\
\text { (Mission) }\end{array}$ & \multicolumn{1}{|c|}{ Channels } & $\begin{array}{c}\text { Number of } \\
\text { angular views }\end{array}$ \\
\hline $\begin{array}{c}\text { 3MI (Metop-SG) } \\
{[2]}\end{array}$ & $\begin{array}{l}\text { VNIR : 410, 443, 490, 555, 670,765, 865, 910 nm } \\
\text { SWIR : 1370, 1650, 2130nm }\end{array}$ & $>14$ \\
\hline $\begin{array}{c}\text { SPEXone (PACE) } \\
{[3]}\end{array}$ & $\begin{array}{l}\text { Spectral resolution is 2-5nm for radiance, 10-40nm } \\
\text { for DOLP, over spectral range 385-770nm }\end{array}$ & 5 \\
\hline MAP (CO2M) & $\begin{array}{l}410,443,490,555,670,865 \mathrm{~nm} \\
+ \text { cross-calibration/co-registration band at 763nm }\end{array}$ & 5 \\
\hline
\end{tabular}

Since an important goal of the instrument is to provide aerosol correction factors for the $\mathrm{XCO} 2$ retrieval performed by the main CO2I spectrometer, the MAP across-track swath has to be at least as large as the CO2I swath, namely $\sim 300 \mathrm{~km}$. This leads to an instrument with large effective footprint - at the mission altitude of $\sim 735 \mathrm{~km}$, the required views are spread out over $\sim 2000 \mathrm{~km}$ along-track by $300 \mathrm{~km}$ across track.

\section{Footprint of view centres}

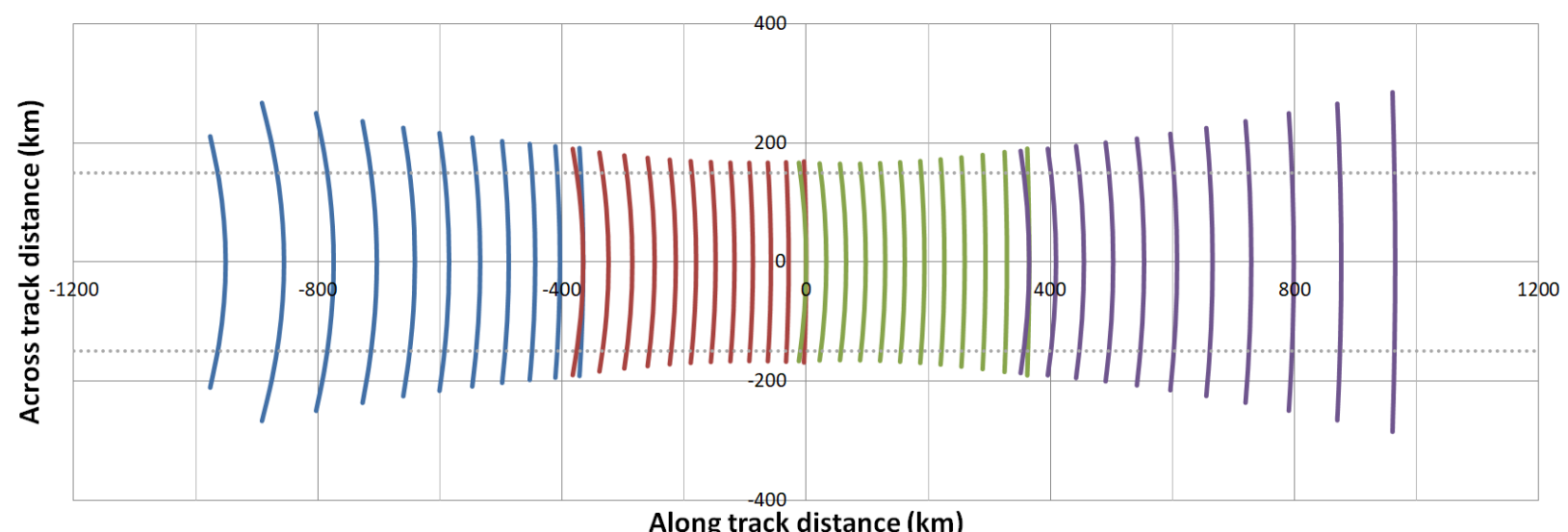

Figure 1. Projection of MAP views on ground, showing footprint is effectively $2000 \mathrm{~km} \times 300 \mathrm{~km}$. Views are coloured by camera number, dotted grey line shows required swath. Overlap of views between cameras can also be seen. 
The driving retrieval requirements are that the retrieved DOLP precision error has to be $<0.0025$, and the systematic error also $<0.0025$, both at $99.7 \%$ confidence. Both of these requirements are very challenging to meet. This requirement is applied at a L1 product level, averaged over $4 \times 4$ spatial samples; the size of the spatial samples - i.e. instrument spatial resolution - is separately required to be $<1 \mathrm{~km}^{2}$.

The precision error requirement leads to an instrument requirement to minimize the effect of all noise sources, principally photon noise. Although this can be achieved simply with large instrument aperture, the need to remain a compact instrument dictated use of a detector which could support a high frame rate - to allow large amounts of oversampling - while having a high intrinsic well depth and large numbers of pixels to support binning. The selected detector was the Teledyne e2v CIS120 device (see later).

The low systematic error leads to a requirement to minimize straylight wherever possible, a historic problem for instruments with large fields of view. Multiple optical configurations, both refractive and reflective, were evaluated during the Phase A/B1 activity to address this. The low error also dictates a system design in which ageing of polarization performance over life has to be minimized. Finally, it dictates that the ground characterization/calibration is performed to very high accuracy, and dedicated on-ground calibration facilities are being designed to provide this.

\section{INSTRUMENT DESIGN}

\subsection{Focal Plane Assembly}

The core innovation for the MAP instrument is in the Focal Plane Assembly (FPA), which consists of a Teledyne e2v detector, a micropolarizer from Moxtek (US), and a spectral filter from Optics Balzers (DE).

The heart of any instrument is its detector, and for this the CIS120 CMOS detector has been chosen. The CIS120 product family has been developed by Teledyne e2v as a general purpose imager for space applications with design features implemented such that it is considered radiation hard. The CIS120 provides a unique combination of a back-thinned imager with on-chip ADC and LVDS digital output making it suitable for use in space. Among its features, the following are relevant for use in MAP

- CMOS image sensor with 2048 x 2048 pixels in a $20.48 \mathrm{~mm}$ square image area (pixels are 10um x 10um)

- Several modes of operation : rolling shutter (RS), global shutter (GS), including on-chip CDS for RS or external DDS for low readout noise

- Configuration, including shutter mode, ADC resolution (8-14 bits per pixel), line pixel address, bias values, programmable by serial input

- High well depth of $\sim 80$ ke-; back-illuminated sensitivity and spectral response

- Pixel access per-row, allowing a pixel mapping to be used to skip unwanted rows, improving effective frame rate

Finally, the same detector is chosen for the VIS and VNIR channels of the main NO2/CO2 spectrometer on CO2M, allowing programmatic efficiency.

A micropolarizer is used to perform spatial separation of the polarized light incident on the FPA. This is a wire grid polarizer from Moxtek, manufactured and patterned using electron beam lithography. It is patterned so that it consists of 10um pitch rows of polarizer alternating between $0^{\circ}, 60^{\circ}$ and $120^{\circ}$ degree orientation. This is aligned and bonded to the silicon of the detector with sub-micron accuracy, and essentially splits the focal plane into three interleaved detectors of $2048 \times 682$, each measuring a different polarization. This is an adaptation of an existing product where pixelated micropolarizers are used to split the light into 4 polarization states at the pixel level; for $\mathrm{CO} 2 \mathrm{M}$, row-orientation is adopted because the instrument is already operating in a push-broom mode. Moxtek wire-grid polarizers are well known, and are also flying on the JPL MAIA instrument.

The relative alignment of the micropolarizer to the underlying pitch of the detector is critical to the performance of the instrument. Teledyne-E2V heritage from previous developments in which direct attachment to the silicon retina is used, allowing the glue line between the polarizer and the silicon to be minimized. The back-illuminated nature of the detector 
means that the pixel reticulation is not visible, and so an active technique in which alignment is performed on an operating detector illuminated with polarized light was successfully developed by TE2 $\mathrm{V}$ as part of the predevelopment activity.

Spectral separation is performed via a dichroic filter developed by Optics Balzers. This consists of $\sim 150$ stripes of single spectral bands, cycling through the six channels. Although each detector is only required to provide 12 downloaded views - where a view consists of one set of the 6 channels - this oversupply allows the selection to be adjusted post assembly on the basis of defects. The additional $753 \mathrm{~nm}$ channel is located at the edges of the filter, in order to provide a coregistered band with the nadir pointing $\mathrm{NO} 2 / \mathrm{CO} 2$ spectrometer.

The filter itself is lithographically patterned, and built up in several coating runs. This approach was selected rather than the alternative so-called "butchers block" because the loss of detector real estate caused by block glue lines could not be afforded. Typically this results in poorer spectral performance due to limitations associated with patterning, but Optics Balzers have developed, as part of the technology predevelopment, an approach which combines the high out-of-band rejection of homogeneous filters with the flexibility of patterning. Spectral separation of the individual bands is enhanced with intervening optically black stripes.

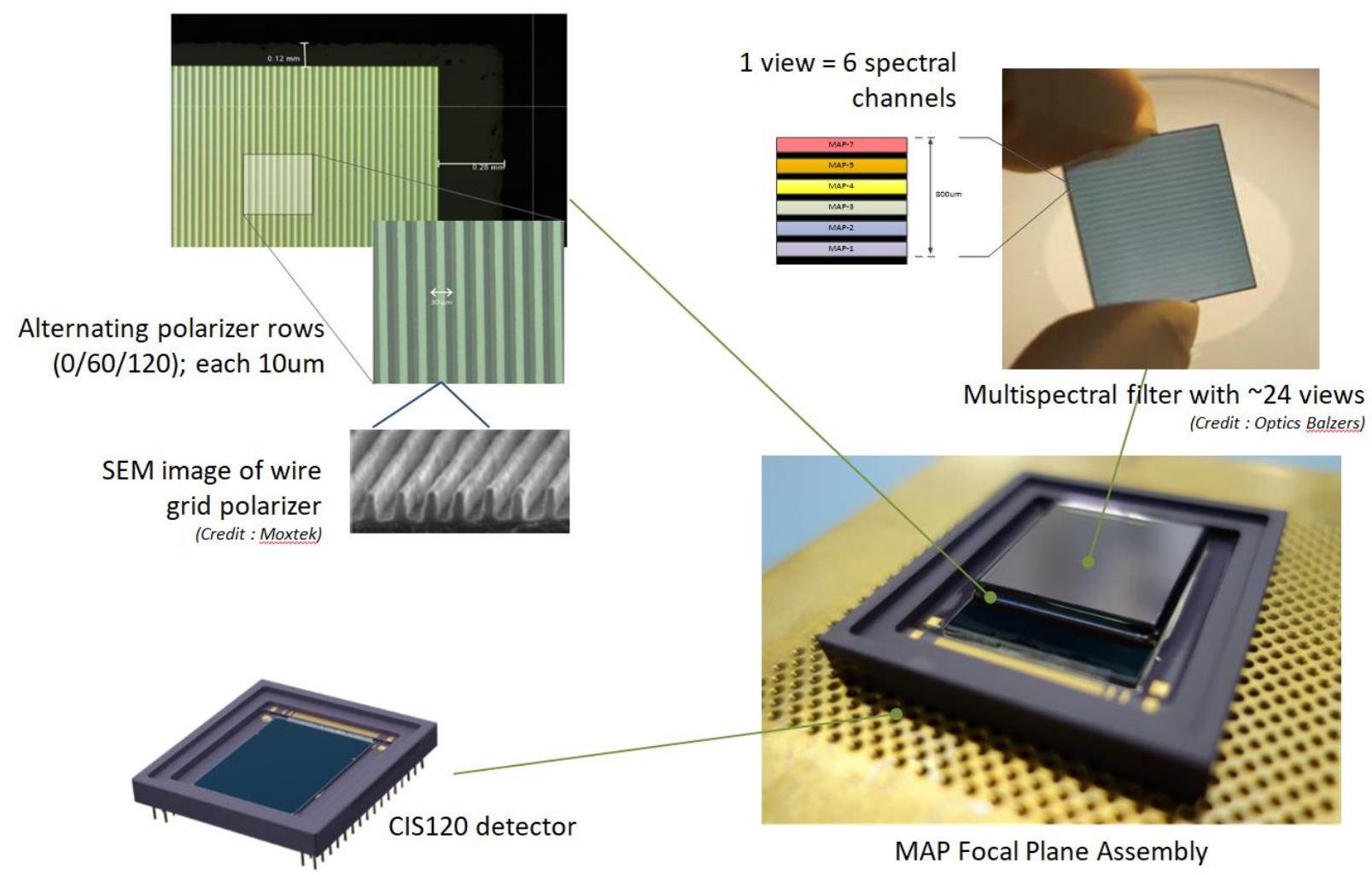

Figure 2. Focal Plane Assembly, showing how a polarizer and filter are combined on top of the CIS120 detector die

\subsection{Camera design}

The field of view is divided between four identical cameras, where each camera includes a separate Focal Plane Assembly. The design of the FPA requires the camera to be telecentric in the image plane. The camera optical design is then optimized for minimum straylight and simplicity. It consists of two spherical mirrors in protected silver, arranged in an off-axis Schwarzchild configuration with accessible pupil, leading to a square field of view of $\sim 28^{\circ} \times 28^{\circ}$. The distortion associated with spherical mirrors is acceptable for this instrument, while the sub-nm surface roughness easily achievable with spherical mirrors reduces the diffuse straylight. The accessible pupil allows for a stop to further manage the straylight. 
The F-number of the design is dictated by the vignetting requirements of the filter, and has to be slower than F/17. This has benefit for the straylight management as the system can be well baffled between M2 and the focal plane, and also simplifies assembly and alignment. The telescope aperture results in a diffraction limited system at focus, but additional defocus is applied in order to widen the point spread function, minimizing aliasing effects when the MAP product is resampled to the $\mathrm{CO} 2 \mathrm{I}$ spectrometer product grid.
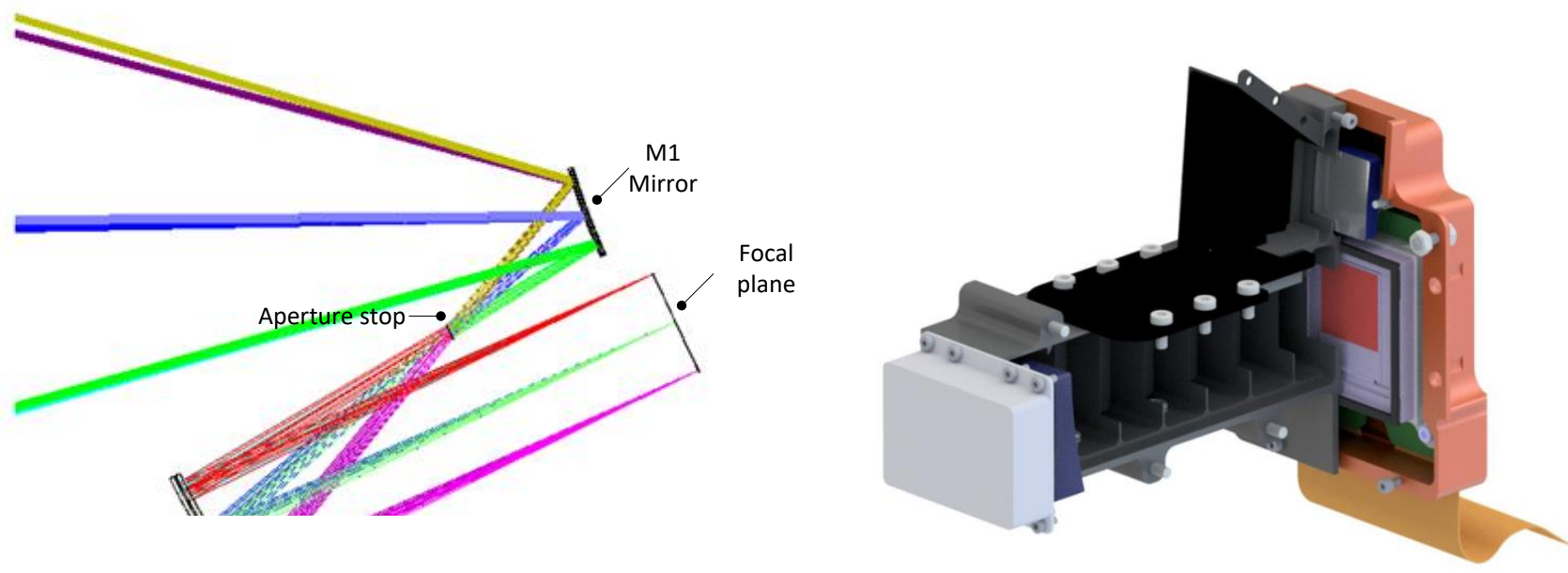

Figure 3. Optical layout (left) and section of mechanical design (right) of single MAP camera, showing off-axis spherical mirrors, and telecentric image plane.

The optical and mechanical parts of the cameras are manufactured by Optec S.p.A (IT). Active thermal control is applied to the cameras to minimize thermoelastic changes in pointing, but also to minimize potential changing stressbirefringence effects in the focal plane.

\subsection{Retrieval}

In order to reduce the data volume downloaded to ground, pixels are binned on-board, in groups of 4 , following gain correction. The detector is run at $\sim 80 \mathrm{fps}$; the CIS120 allows sufficient control of its sequencer that readout of only the rows that are illuminated can be performed, which allows the apparent frame rate to be high. Multiple frames are then combined on-board to reduce the product rate to $\sim 8 \mathrm{fps}$. Since the instantaneous footprint of the three rows required to recover the DOLP of the scene are separated, the start of the oversampling point is offset between each row, to reduce the spatial error of the polarization recovery.

All this processing is performed by FPGA within the instrument electronics unit.

\subsection{In-flight Calibration}

Degradation of the optical performance over life is minimized by design, principally through use of radiation resistant fused silica for the transmissive elements, and avoidance of molecular contamination sources. Nevertheless, loss of throughput, especially in the blue channels, is expected due to the mission life (7 years), and the UV browning of residual organic contaminants. The MAP instrument and $\mathrm{CO} 2 \mathrm{M}$ mission allow for multiple routes to tracking and correcting for this degradation. 
Each camera is combined with an individual on-board mechanism which allows the insertion of transmissive diffusers into the optical path, for calibration using direct solar illumination. This system is built by Micos $(\mathrm{CH})$ and is based on similar systems in the UVNS instrument on Sentinel 5. No modification of mission attitude is required, since the mission uses a particular sun-glint-tracking orientation over the oceans which helpfully puts the sun in the camera field of view at higher latitudes.

Cross-calibration between the nadir-pointing views of MAP and the NO2 spectrometer on board $\mathrm{CO} 2 \mathrm{M}$ is possible over the 410-490nm channels; additionally the $753 \mathrm{~nm}$ channel is located in these views allowing cross-calibration with the NIR band of the main spectrometer. This also provides a reference band for certain vicarious calibration approaches expected to be deployed.

The orbital geometry - sun-synchronous orbit with 11:30 descending node time - also permits the moon to be used as a stable radiance target during eclipse. Once every 2 months the spacecraft performs a pitch manoeuvre in eclipse which allows the moon to be scanned across the field of view of all 4 MAP cameras. Once corrected for phase and libration, this is expected to provide a highly accurate ageing reference, as well as a reference to correct for pointing bias change over life.

No on-board polarization calibration source is included, since the principle source of polarization modulation - the micropolarizer - is well protected and intrinsically stable.

\subsection{Instrument architecture}

Each camera includes further baffling to minimize out-of-scene straylight, and in particular to minimize the effect of the sun when the inside edge of the baffling is illuminated by the sun during a sun-glint tracking pass.

The four cameras are arranged adjacent to each other on an optical bench, with their boresights fanned out in the alongtrack direction. The optical bench is cold biased via a radiator on the shadowed side of the spacecraft; this provides the cold sink for the active thermal control to work against.
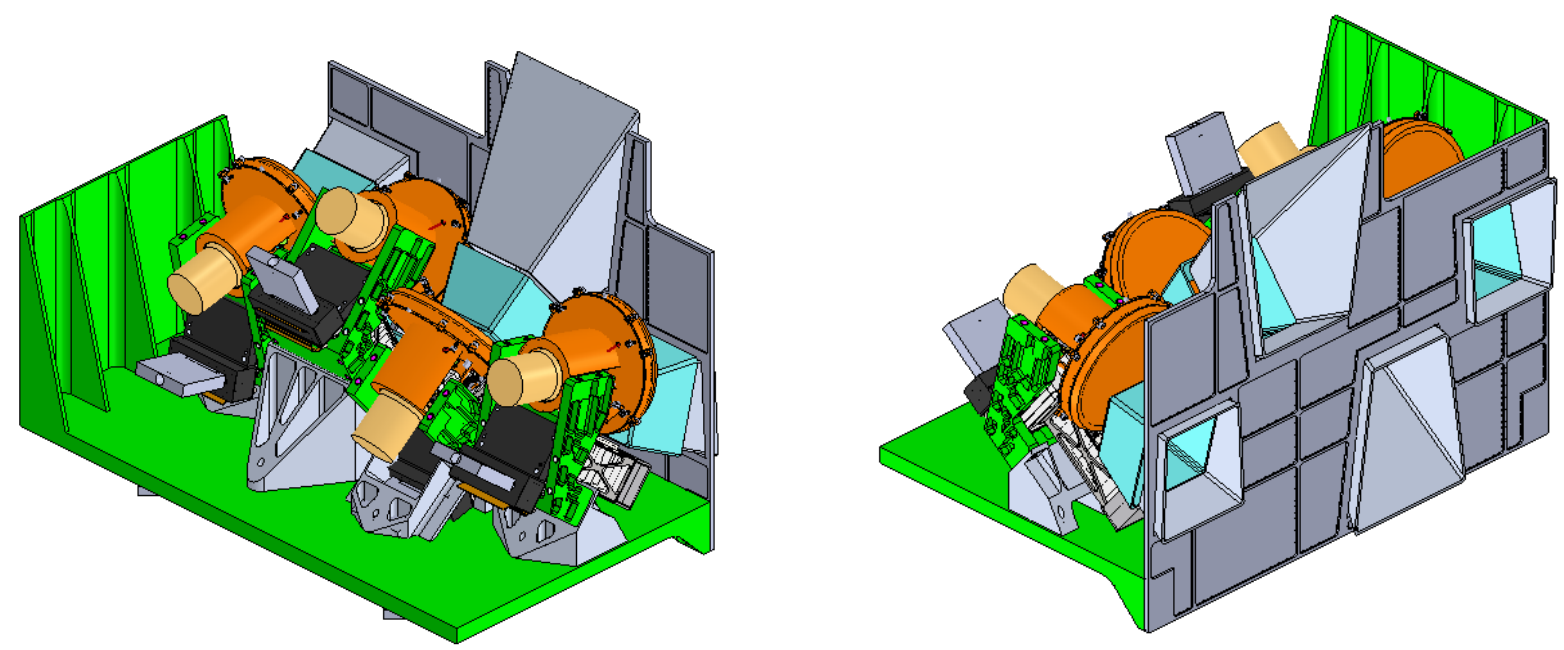

Figure 4. MAP Optical Unit (in development), showing arrangement of four identical cameras, calibration subsystems. Housings, MLI, harness etc. removed for clarity.

The optical unit is quite small $(540 \times 380 \times 330 \mathrm{~mm})$ and light $(\sim 12 \mathrm{~kg})$. It is supported by a separate electronics unit which contains the bulk of the processing electronics. 


\section{CURRENT STATUS AND RESULTS}

The programme is in phase B2, with instrument Preliminary Design Review (PDR) due to take place in late 2021. The overall schedule is very demanding, with qualification models of the optical system commencing testing very soon after PDR.

The predevelopment of the FPA has completed with the production and characterization of the performance at FPA level already completed. These FPAs are being incorporated into telescopes in Q1 2021, with performance measurement at camera level being performed in Q2 2021. Environmental qualification, including radiation testing, of the detector is nearly complete.

A critical aspect of the instrument performance is the performance achievable by the FPA. In particular, polarization "cross talk" can occur between adjacent rows, which degrades the ability of the detector to resolve the DOLP of the scene. This can arise from a number of sources, for example : misalignment of the polarizer, too large a glue line between polarizer and detector surface, poor polarizer contrast, scatter from either the detector surface or the rear polarizer surface, detector modulation transfer function, etc. This effect has been - and continues to be - a predominant concern. However it is very difficult to model accurately, and there is little data in the literature for actual performance of these type of assemblies, partly because it is very dependent on the details of the assembly. Consequently significant emphasis was placed on direct measurement of the total combined effect as part of the predevelopment activity.

Briefly, a test campaign was performed by Teledyne e2v in which the Mueller matrix of each illuminated pixel in the FPA was established by presenting the FPA with a beam of collimated, fully polarized, light. Rotation of the polarization angle of the incoming light and recording the resultant detector signal results in responses that vary sinusoidally with the polarization angle (according to Malus' law). Numerical fits are then applied to these measurements to determine the apparent extinction ratio and angle of each pixel, which allows the linear terms of the Mueller matrix to be populated. Circular polarization is ignored.

The efficiency of the FPA in acquiring the DOLP is best expressed through the extinction ratio, or contrast, which is the ratio between the signal when the incoming polarization state is aligned with the polarizer - resulting in maximum transmission - and the signal when the incoming state is at $90^{\circ}$ to that state. Any polarization leak results in a lowering of this effective contrast. The results from the FPA measurement show contrast values between 4 and 12 depending on wavelength, which is sufficient for the instrument to be compliant.

The underlying polarizer itself has much higher contrasts $(>200)$, and the separation between polarizer and detector between 5-8um - coupled with the illumination geometry and predicted detector reflectivity in index matched adhesive would suggest a higher contrast should be obtained. However, in this application, the dominant performance term is believed to be the detector MTF, because, effectively, polarized light passing through the micropolarizer appears to the detector as a modulating signal at $2 / 3$ of the Nyquist frequency; the performance then follows the predicted detector MTF quite well. Using a back-illuminated device gives better MTF performance in the NIR than the UV due to the depth of the generation of the electrons in the silicon, and it is interesting to note that a change to a front-illuminated device in the future would most likely improve the UV retrieval, which may be of interest for instruments focusing on PM2.5 type aerosols.

The spectral response of the filters was also measured during the predevelopment and found to be satisfactory. 

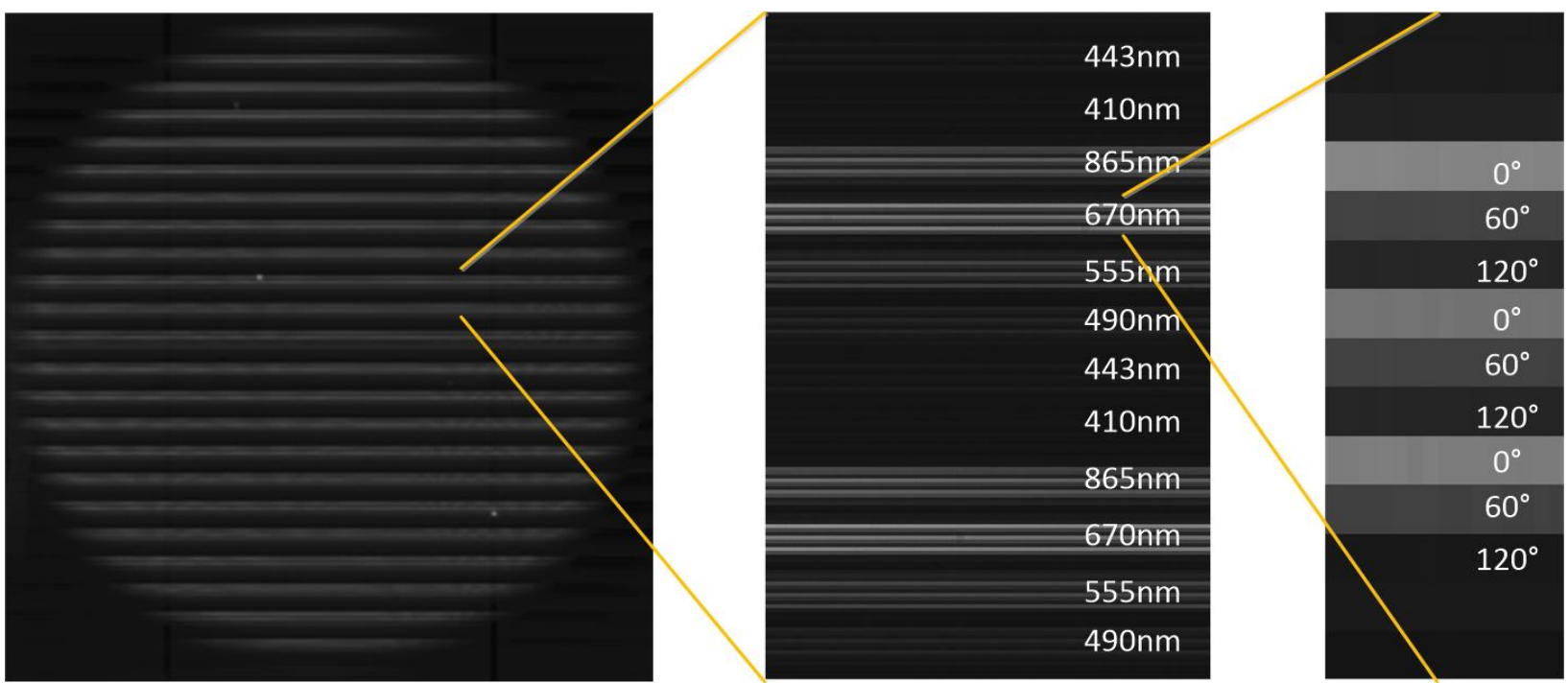

Figure 5. (Left) Image taken from trial detector when illuminated with broadband polarized illumination from a QTH lamp, showing variation in each band due to spectral sensitivity and lamp output. (Centre) Detail showing 2 views covering 6 spectral bands (Right) Detail showing varying polarization response from the adjacent rows within the band

\section{FUTURE ACTIVITES}

In addition to use on the $\mathrm{CO} 2 \mathrm{M}$ mission, the use of compact polarimeters for aerosol retrieval over cities from cube or microsatellites is currently being studied by TAS, as part of a UK funded activity with the University of Leicester and DOrbit. In this application the agility of a cubesat is used to remove the need for multiple telescopes, as it can scan a single MAP-type focal plane over a city dynamically. Aerosol retrieval in this context can be aimed both at health applications, monitoring adherence to pollution goals, as well as climate generally.

The design of the focal plane in which spectral, polarization and spatial separation is performed by lithographically patterned items, allows great flexibility in customizing the approach to different applications. The existing MAP FPA with 24 angular views - allows very good imaging of the 3D structure of aerosol plumes or cloud tops, which includes the possibility of determining wind vectors.

\section{ACKNOWLEDGEMENTS}

The development of this instrument has been entirely funded under ESA contract.

\section{REFERENCES}

[1] Bernd Sierk, Jean-Loup Bézy, Armin Löscher, Yasjka Meijer, "The European CO2 Monitoring Mission: observing anthropogenic greenhouse gas emissions from space," Proc. SPIE 11180, International Conference on Space Optics — ICSO 2018, 111800M (12 July 2019); https://doi.org/10.1117/12.2535941

[2] Ilias Manolis, Jérôme Caron, Semen Grabarnik, Jean-Loup Bézy, Maurizio Betto, Hubert Barré, Graeme Mason, Roland Meynart, "The MetOp second generation 3MI mission," Proc. SPIE 10564, International Conference on Space Optics — ICSO 2012, 105640A (20 November 2017); https://doi.org/10.1117/12.2309178

[3] Aaldert van Amerongen, Jeroen Rietjens, Jochen Campo, Ersin Dogan, Jos Dingjan, Raj Nalla, Jerome Caron, Otto Hasekamp, "SPEXone: a compact multi-angle polarimeter," Proc. SPIE 11180, International Conference on Space Optics — ICSO 2018, 111800L (12 July 2019); https://doi.org/10.1117/12.2535940 